\title{
Presentación
}

\section{EN DEFENSA DE LOS DERECHOS HUMANOS. ACTUALIDAD DE IGNACIO ELLACURÍA}

La actualidad de Ignacio Ellacuría se basa en haber conjugado el análisis político de la realidad, el quehacer universitario, y la reflexión filosófica con el compromiso vital -personal y comunitario-.

De su primera etapa, etapa de formación y estudios, anterior a la influencia directa de Zubiri, cabría destacar sobre todo el sentido de la historicidad transmitido por Karl Rhaner y recogido en buena parte en la Constitución sobre la Iglesia en el mundo actual (Constitución del Concilio Vaticano II, promulgada el 7 de diciembre de 1965).

La dignidad de toda persona humana, el sentido de la comunidad, el compromiso como praxis transformadora, el análisis de la vida económica-social, la vida en la comunidad política, la autodeterminación, el fomento de la paz y la no-violencia, y la promoción "comunitarista" de los pueblos en un mundo global, son aspecto claramente asumidos en esta primera etapa.

De hecho, Ellacuría, tras su licenciatura en Filosofía (en 1955) en Quito, y su actividad como formador, entre 1955 y 1958, en el Seminario de San José de la Montaña (San Salvador), marcha a Innsbruck (Austria), donde estudió Teología. Pero descarta realizar una Tesis Doctoral en Teología sobre la historicidad de la idea de Dios. De 1962 a 1965 se traslada a Madrid y realiza sus estudios para el doctorado en Filosofía, en la Universidad Complutense. En Madrid, tiene una gran acogida en casa de un hermano suyo,
Juan Antonio, quien le facilita un despacho y el compartir su vida familiar.

La incidencia de Zubiri va pareja con la exigencia de la restauración de la vida intelectual y el quehacer del "filo-sofo", amigo del saber más real de la realidad, que ya quedaba clara en el libro Naturaleza, Historia, Dios, en el que -con motivo de estudio de "Hegel y el problema metafísico"- Xavier Zubiri también planteaba una reflexión sobre realidad e historicidad.

Su Tesis Doctoral en Filosofía (de 1965, en la Universidad Complutense de Madrid) Ileva por título: La principialidad de la esencia en Xavier Zubiri.

En 1967 regresa a El Salvador para incorporarse a la Universidad Centro Americana (UCA) "José Simeón Cañas" como profesor. Mantiene la colaboración con Xavier Zubiri y viaja a menudo a España. Pero la Conferencia de Medellín (II Conferencia del Episcopado Latinoamericano, de 1968) marca también su reflexión y producción teológica orientada hacia la liberación.

Desde 1968 hasta su muerte será miembro del equipo rectoral, denominado "Junta de Directores" de la Universidad de la UCA, de la que será un cualificado motor incluso antes de ser el Rector. Ya en 1969 logra que la UCA asuma la revista de Estudios Centro Americanos (ECA), en la que publica muchos de sus artículos filosóficos, teológicos y políticos.

De 1970 a 1973 se hace responsable de la formación de los jóvenes jesuitas de la 
Provincia Centroamericana, cargo que le lleva a conocer al padre Arrupe, General de la Compañía, defensor del principio de la encarnación en el trabajo pastoral, con quien siempre mantendrá una relación de afinidad.

En 1972 es nombrado Director del Departamento de Filosofía (pues, la UCA no tiene Facultad de Filosofía), y en 1973 publica su libro Teología política, obra que será editada posteriormente en inglés, en Nueva York, en 1976, bajo el título Freedom Made Flesh: The Mission of Christ and His Church.

En 1974 funda el Centro de Reflexión Teológica en la UCA. Al año siguiente participa en el homenaje a Karl Rhaner, sintetizando en un ensayo las denominadas Tesis sobre posibilidad, necesidad y sentido de una Teología Latinoamericana.

En 1976 es nombrado director de la Revista de Estudios Centroamericanos (ECA). La publicación del famoso editorial: "A sus órdenes, mi capital", parece que ocasionó la retirada del apoyo económico del Gobierno salvadoreño a la UCA, provocando además una clara violencia paramilitar contra la Universidad, a la que siempre Ignacio Ellacuría quiso autónoma, respecto del poder civil y del poder eclesiástico. Sobre todo a partir del año 1979 en el que es nombrado Rector, dos años después del asesinato del P. Rutilio Grande cuando Ellacuría estaba en España.

El hecho sacudió espiritual e intelectualmente a la comunidad de los jesuitas y produjo una transformación interior en la persona del arzobispo Óscar Romero. Ellacuría vive su "primer destierro". Desde el 12 de marzo de 1977 todos los jesuitas son amenazados de muerte. Ellacuría volvió a El Salvador en agosto de 1978.
En 1979 se produce un Golpe de Estado de la Junta de Gobierno en El Salvador. Fracasa este intento y se desencadena una cruel violencia y guerra en el país. En 1980, el 24 de marzo, es asesinado el arzobispo Mgr. Romero durante la eucaristía. Y, a finales de ese mismo año de 1980, Ellacuría sale de nuevo, esta vez "desterrado" a España, bajo la protección de la Embajada Española. Desde entonces, Ellacuría aprovecha los viajes a España para dejar oír su voz en Europa, DEFENDER LOS Derechos Humanos, y activar la publicación de algunas obras de Zubiri, con quien compartió, más allá de la Ontología, el que es la realidad compleja, dinámica y abierta el objeto de la Filosofía, en tanto que realidad histórica no factum sino como faciendum.

Entre 1980 y 1983 se publica la trilogía de Zubiri, X.: Inteligencia sentiente (Alianza Editorial, Madrid 1980), Inteligencia y logos (Alianza Editorial, Madrid 1982), e Inteligencia y Razón (Alianza Editorial, Madrid 1983). Desde 1980, El Salvador vivirá una larga guerra civil de doce años, en los que la Guerrilla se enfrentará permanentemente al Ejercito Nacional. Y ya en 1981 Ignacio Ellacuría planteó abiertamente la solución dialogada-negociada al conflicto.

En diciembre de 1982 con motivo del aniversario de la Declaración Universal es invitado por la Asociación pro Derechos Humanos de España a impartir una conferencia en Madrid. El documento manuscrito que aportamos es inédito. Constituye el primer borrador de la misma, que juzgaría muy extensa y que acortaría a posteriori. El segundo documento -mecanografiado- es el corpus completo de su ponencia. A partir de ella se publicaron dos artículos de opinión en el diario El País bajo el título: "Los Dere- 
chos Humanos en Centroamérica" (I y II parte, los días 23 y 24 de diciembre). En este discurso Ellacuría pide a España su mediación entre Europa y la comunidad latinoamericana. Le insta a no permanecer neutra ante la injustica estructural y le sugiere ser adalid de los Derechos Humanos ante la comunidad internacional. Un llamamiento que sigue resonando en nuestros días.

Tras la muerte de Zubiri (año 1983), Ellacuría es nombrado Director del Seminario Xavier Zubiri.

El año 1984 publica en España un libro que interpela a la Iglesia institución: Conversión de la Iglesia al Reino de Dios. Ese mismo año, con Jon Sobrino funda la $R e-$ vista Latinoamericana de Teología.

En 1985, con monseñor Rivera y Damas actúa de mediador para lograr la liberación de la hija del Presidente Duarte, secuestrada por la Guerrilla, y de 22 presos políticos. Y, en 1986, sigue insistiendo en la necesidad de una salida negociada y dialogada al conflicto civil de El Salvador.

En España, con motivo del primer Encuentro de Religiones Abrahámicas celebrado en Córdoba, en 1987, expresó públicamente la necesidad de encontrar una perspectiva y bases comunes para superar positivamente los conflictos. Su ponencia en aquel encuentro fue un "Aporte de la Teología de la Liberación a las Religiones Abrahámicas en la superación del individualismo y el positivismo". De hecho, ese mismo año durante el verano, con motivo de unas Jornadas de reflexión y diálogo celebradas en la Biblioteca de la Universidad Iberoamericana de La Rábida (Huelva), sobre las implicaciones sociales y políticas de la Teología de la Liberación, Ignacio Ellacuría volvería a reiterar públicamente la misma idea, instando a la reconciliación, con el fin de hacer posible un proceso de democracia real y de convivencia pacífica.

En aquellos momentos, España y Europa eran el lugar idóneo para expresar en alta voz su pensamiento, con el fin de que llegara a los Centros de decisión y de poder Internacional.

A primeros de noviembre de 1989 Ellacuría recibía en Barcelona el Premio de la Fundación Comín, otorgado a la UCA de San Salvador. Mientras, el Gobierno de aquel país temía no poder frenar la presión de la Guerrilla en la propia capital de San Salvador. Ellacuría adelantó su regreso a El Salvador sobre el 13 de noviembre, para intentar mediar una vez más en pro de la paz y la convivencia. Pero, como altavoz de la Teología de la Liberación, dado su prestigio intelectual y su valiente denuncia de la situación del país, como defensor de la liberación del pueblo y de las mayorías populares, se había granjeado la enemistad de algunos sectores financieros y militares que le amenazaron con insistencia para callar su voz.

El 16 de noviembre de 1989 fue asesinado por soldados salvadoreños del propio Ejército Nacional, en la residencia de la Universidad, junto con los jesuitas Ignacio Martín Baró, Segundo Montes, Armando López, Juan Ramón Moreno, Joaquín López y López. Fueron también vilmente asesinadas Elba Julia Ramos, persona al servicio de la Residencia, y la hija de ésta, Celina, de 15 años. En la actualidad, el cuerpo de Ignacio Ellacuría yace enterrado en la capilla de la UCA.

Su obra Filosofía de la Realidad histórica ha sido publicada post mortem por el profesor de Filosofía Antonio González. En ella queda patente "a modo de conclusión" que "la verdad de la realidad no es 
lo ya hecho; eso sólo es una parte de la realidad. Si no nos volvemos a lo que está haciéndose y a lo que está por hacer, se nos escapa la verdad de la realidad..." El texto reproduce lo dicho en un ensayo de Ellacuría sobre "el objeto de la Filosofía". Para Ignacio Ellacuría "hay que hacer la verdad... hacer aquella realidad que, en juego de praxis y teoría, se muestra como verdadera".

Todo un reto de gran actualidad tanto en el contexto de América Latina como en el conjunto de la Comunidad Humana, en un Mundo Global.

En cuanto al tema de la defensa de los Derechos Humanos, es también muy de tener en cuenta la obra del profesor Senent de Frutos, Juan Antonio: Ellacuría y los derechos humanos. Bilbao: Desclée de Brouwer, 1998. Muy importante, además, el libro del que es editor Juan Antonio Senent: La lucha por la Justicia. Selección de textos de Ignacio Ellacuría (1969-1989), Universidad de Deusto, Bilbao, 2012.

En diciembre de 1982 con motivo del aniversario de la Declaración Universal es invitado por la Asociación pro Derechos Humanos de España a impartir una conferencia en Madrid. El documento manuscrito que aportamos es inédito. Constituye el primer borrador de la misma, que juzgaría muy extensa y que acortaría a posteriori. El segundo documento -mecanografiado- es el corpus completo de su ponencia. A partir de ella se publicaron dos artículos de opinión en el diario El País bajo el título: "Los Derechos Humanos en Centroamérica" (I y II parte, los días 23 y 24 de diciembre). En este discurso Ellacuría pide a España su mediación entre Europa y la comunidad latinoamericana. Le insta a no permanecer neutra ante la injustica estructural y le sugiere ser adalid de los Derechos Humanos ante la comunidad internacional. Un llamamiento que sigue resonando en nuestros días

Sevilla, a 11 de noviembre de 2021

José Mora Galiana

Asociación IESMALA, Sevilla, España jmorgal2@upo.es

\author{
Álvaro Rodríguez Camacho \\ Universidad Pablo de Olavide, Sevilla, \\ España \\ arodcam@upo.es
}


LOS DERECHOS HUMANOS EN CENTROAMÉRICA (NOTAS MANUSCRITAS)

Ellacuría, Ignacio

Los Derechos hurneros en Centrovenerce

1. A peser de sn pequeirey of reletive poce importienase. CA es un lingor hutionio esencial pare la ternia $g$ la reabidad de los $\mathrm{IH}$

- Il hecho cartinimedo de le alenciri mur. chal sube el teme es le mejor pruets

- La explicacion de porgi en CA se da una tal volacine dle JH z une tal exigence de su cuplininto ol exfuerzo por cadbr on a quelle $j$ poh. hlitar éle dou by sohe uno de bi protlenes captiale de mesno tierpo I del mivel itico-politio de le huremidad

+ qravimas z necions nolecuras de bs D H thay eu mu chas destes.

+ Lo $q$. vecurse en C.A es 9 . Te den liternecing hay whe liche

x unjurtica estructural gerere. bi vede if solnece ponlle le vide dil. majistos puiloses

x respuerta politien mulctar de eres maprios

Revista Internacional de Pensamiento Político - I Época - Vol. 16 - 2021 - [633-652] - ISSN 1885-589X

637 
(2)

$x$ aplestameilo susteralue de tre reypmenta q. legis de apregorte whe cale vey Major fuerze + entor válido de Guaterale 2 El sehrachr cre lo fré aute de Nice. rague. I w lo es for dutuntos ra zones de Honduns. Guta Ricia 7 Pare

2. Hug sin pocos las q. wo ven 9. el prothe rachice pricipalun. en be injustecre estors Aurail $f$ en he burquede de une respues? ta eficaz

- Reagan $z$ los sugos sigran repities do 9. el protlere es fundenentalin. el 9. "agente de le inertalihidad dereen impmer mens forrs de

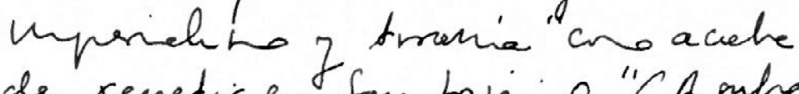
de repetior en San tose; O "CA ruple les cruseancis de une eerrom. arretecle $f$ une infurrecain apryas da por fiverzas externs" (Broguta)

- Betoncur en conhis, hablo de "hogue ras prenchdos por le unpustices socal - por nernos extoarios an taleg mos" dicendo 9. mis 9. un problene de subverni es un priblene de rubdere.

Revista Internacional de Pensamiento Político - I Época - Vol. 16 - 2021 - [633-652] - ISSN 1885-589X

638 
3

- duen Pall II he heelo dos forinba wes erenciales she be violencre en ES.

+ "me diy perfectam. cuenta de p los

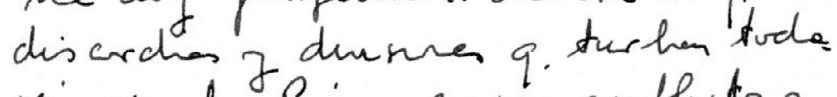
vie vrestro Pais center crifh tis 2 violencas, enculoulron th raiz verclads ra en las situearias de unjuntica bocial"

+ en le luche fratricide a un la do entain " cuentos curnderon le luche ormeda ans un untrusento mecere tio pare conkegirs un mueno orden sociel. I al otro lacb a cantos oe curren a los pmicypis d l 'segurictad macenel pare legitivior reguenis buitales"

3. La prerencie forminea en C.A. g. es especalun de USA subroline tode politice de Dit a la polikiea delmen

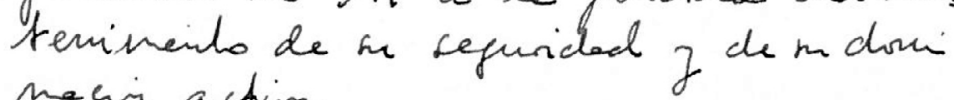
mecin active

- USA querie termio un la vola ani d'l. DH cr tal d.q. ente terranito no sumpiene in arrence d l fuerges reviluciones

+ lo he hecho cuendo eitos ro eran villentos: Arlenz. Bosch. Allende 7 hohio gando densidien.

Revista Internacional de Pensamiento Político - I Época - Vol. 16 - 2021 - [633-652] - ISSN 1885-589X

639 
(4)

+ lo hece ahrie en Nicoregre escialos do qrechalm. in violencise crilre elle

+ Lo hizo un ES cuando los aresunetos supencher los 10.000 al aino $z$ lo $f$. gre hecendo

+ lo ve a hecer ahore on Ris Munt a pesor de le harhorie del meno ré given

- Esta subordinicin d l. DH a razmas de preder $f$ domiecin hace 9. algn. nos de sur presing position en farr d l. DH presdan vatidey etive pwhitie o am eficecia o unim pere be sols anies reeles

- Se ve cro recesoria la jualrica. ciri por valores $y$ derectos ture. nos peno te sonde lo hureno a lo polition g eevino

+ basta cris un peligir para el orden establecido miteno unterecinal pere q. Is poleres interor a niteracinale crrerpelen al himbe $z$ sus derectos

+ el seenfino gerocide de nds tumaras no le a cergunento pore canch de politica

* Israel

x guterale a ES

Revista Internacional de Pensamiento Político - I Época - Vol. 16 - 2021 - [633-652] - ISSN 1885-589X

640 
(5)

4. ¿ He halido mejore en el rerpeto a bs DH en ES g Tuaterale duronte 1982?

- La cuertion refende a le sitmecirn real de ES $z$ queternala carece de sentrido en el plars de los DH

+ el aumenb o dismicari anarlsta. tive $d$ l.aresinetos anoudo be cor lided mine $g$ le anahided in nitolerelles es un puso prege polite. Co q. Meche hene q. ver on bi DH

+ Reigar cerlaficara la mejone sies 9 . le mecesita pase el cry trol nulitor de le zuna 7 para eno presurara for nejorns a futuro q. permt on borasseguir d l. Dit protende on be volacior

+ lo improstane es juzgor riese "neqive", aung. finere lenta, tiene por objetivo bo DH segin astos ci terios:

$x$ subrodivacirm del a prozedo politice al respeto IH

$x$ q. la volecior 14 no sea par le surtanciel del progectop? litivo-ninlitor

* desnoulelaniento del apraneto represivo + esoro quita pare q. se busquen

Revista Internacional de Pensamiento Político - I Época - Vol. 16 - 2021 - [633-652] - ISSN 1885-589X

641 
(6)

- Los hechos en Ghatende o en ES no per ruten hellor de nejorie porg. los linites
sun nitolenelles

+ en Ghat. tal vez so haye tondo caseminelo politio en le coputal pero los areninetos de le poblecinin undigere se hou sisterahizado $z$ profundizado $x$ no hay politice de lierse qrenecte sno de armenstis que.

* todo presundo simpelizente. fanher o colebondor dl. quenille es cenchidato al are sinelo * te hisce el anigulariento del eferato.

+ en ES ame sigren suido allivios - J smaderin a cunbelivos-bsasen netos:

$x$ "descle 1979, tal vey uns 30.000 salvedoreios hou rido areninados in mados en hetalla. Menos de 1.500 censs... har sido llevados arie los inburals de funteise... Ha helich mers de 200 curclenas por entos criveras (Hintion, 29.0d.82)

Revista Internacional de Pensamiento Político - I Época - Vol. 16 - 2021 - [633-652] - ISSN 1885-589X

642 
(7)

x "Nuertre rerpunalihded de jefes de Eita. do no nos pernite pernowecer npentlos ante le aplesture de foros drarios en el sueb de mertre qeogrefie cormin;30.000 tunhos en ES. pare neuarior we wo necim, secuder les anciei as dinger les achormetides "(Betanceses, 3 dic .82. ane Reegan)

$x$ de Enero a 15 Nov. 1982 he hahdo 4168 cuiles areniredes, he mitod de los hahdos en 1981 0 1980, pero sin ary tor las victivios curlas d liguerna en las culra ofentions

* el ceso de la Corpenative de La Floride (v.documento)

* Liel dereclo a le vida enta en esos andicinas, el resto de IIt casimi es cuestion

$x$ us hey prisirioros de guerne for raste de le FA, aunq. elFMLN the entregeb mis che 200 a la CRI $x$ el decrelo 507 perrite terer sin protecenin legel 120 diras a los mero res de 16 aites 9 ls megres 6 meses

* no hey josthlidael de oporicum politice organgade $z$ es repride le puotesta suichical

Revista Internacional de Pensamiento Político - I Época - Vol. 16 - 2021 - [633-652] - ISSN 1885-589X

643 
(8)

5. ¿ Hoy solucines pare esta situreciir?

- La sulnair por arriqulemieno llave al gernocidis tientoen Gretereb con en ES

t en Ghaterole esta sulncin es le q. prechonie $z$ va en aumento + en ES no se he ahendrodo pero va en disminari

$x$ la guera as cade vez mas fuese (Hindon recurece 4000 bejos en bos 15 ultinos meses en le FA, de los g. os de 1.000 serin Notials): ar le ojernine de Oct. Nov the hatide 210 prisur. 200 mase tos. 343 hendos 7 se han cepturado 422 fisiles, 25 arros de apuje.100.000 cer

$x$ mo se ve le porherded to mediata de amiquelemer.

- La soluair "denocrelizedre" es umpo sille en hi inima sino ve acrupaine de de algue fore de accuedo con los murvinentos revolucurnoros + sir ellos as false porg deje la

Revista Internacional de Pensamiento Político - I Época - Vol. 16 - 2021 - [633-652] - ISSN 1885-589X

644 
(9)

cono gandarne d l. interens morleahe ricaros (Hondernes)

* cr apareincios devocrélicas y sin presin pugular no halor mi siguiere reformes bences $x$ la dependence de USA es tótal peno descle la perpective dl em. puese privede

+ no hey la voluntal en el ceputal de hear posille $z$ lo reupetor bas andicurs a los remettados de une crnelta populor

- La solucir ne gociada puede conseguis al meros un pronbo fir a la gresa sir 9. esto inpligu dismmai de presin por pask del novimerb reveln.

+ la finerze militor 2 prover hece posille un regociacin q. logre renctados selisfectonos dimunento $f$ a futuno

+ mede neutrchizar a los ceurantes mapres d l. virlecui d l.DH grear conchicuris pere in respelo

- La expecial relecuir ES-USA g Guatera. le-USA hace $q_{i} l$. puenin unteracinal en el tere d l. $2 H^{\prime}$ see un arre efective pare in mequienento.

Revista Internacional de Pensamiento Político - I Época - Vol. 16 - 2021 - [633-652] - ISSN 1885-589X

645 



\title{
LOS DERECHOS HUMANOS EN CENTROAMÉRICA (TEXTO MECANOGRAFIADO)
}

\author{
Ellacuría, Ignacio
}

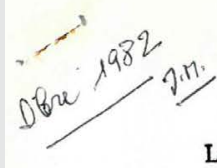

LOS DERECHOS HUMANOS EN CENTROAMERECA

La Asociación pro derechos humanos de España ha programado una Semana por los derechos humanos del día siete al diez dediciembre, cuyo primer acto fue dedicado a "Los derechos humanos en el mundo, båance de un año". Tres zonas ocuparon especlal atención: la que afecta al pueblo palestino, cuya presentación corrió a cargo de Roberto Mesa, Vicerector de la Universidad Complutense: la que afecta al pueblo polaco presentada por Fernando Claudín, director de la Fundación Pablolglesias y la del pueblo centroamericano que me fue encargada a mi. Quisiera con esta ocasión poner por escrito algunas de las ideas que preparé para ese acto, algunas de las cuales expuse mientras que otras se quedaron sin exponer.

Centoramérica constituye un lugar hoy fundamental para la recta visión y la justa solución del problema de los derechos humanos. Paises como Costa Rica Panamá ofrecen una imagen aceptable, aunque lejos de 10 Idea1, de los derechos humanos, porque en ambos paises, si bien de disthnta forma y por diversas razones, se ha atendido a tiempo a las necesidades båsicas de su población. En Honduras no ha estalladors todavía la crisis de los derechos humanos, no porque se haya resuelto ya el problema de las necesidades básicas y de la injusticia estructural sino porque no se ha apoderado de la conciencia popular la situación objetive en la que se encuentra la mayoría de la nación, la situación es objetiłamente pre-revolucionaria y poax eso no ha sido necesanto todaviale entrar de 11 eno en la violación masiva y sistemática de los derechos humanosi sin embargo, la presencia en suelo hondureño de tropas somocistas, de asesores norteameri canos que preparan un lugar seguro que domine los acontecimientos de Nicaragua, Guatemal y El Salvador, y, ültimamente, la presencia del ministro de defensa israeli, el mismo que lanzó la ofensiva contra los palestinos en el Libano, hacen temer que tambiẻn Honduras se vaya a ver pronto inmersa en el mismo torbellino de El Salvador y Guatemala por 10 que toca a la violación de los derechos humanos, de 10 cual ya se aprecian algo mås que Iีndicios. Nicaragua, por su parte, estå en una fase distinta, en la que observadores occicentales pueden apreciar limitaciones importantes a los derechos humanos, sobre todo en el orden politico, pero en la que analistas más conocedores de la pecullaridad de la situación nicaragüease no pueden menos de ver no so̊lo una clara mejoría respecto a lo que oourria en tiempos de Somoza sino además una abismal dife. 
rencia con lo que ocurre en Guatemala y en ER Salvador estando como está Nicaragua acosada, sobre todo por Estados Unidos, para que los sandinistas abandonen el poder, un acoso que encluye la violencia armada, además de la presión económica y politica.

Los casos de Guatemal y El Salvador son, sin embargo, los más dramáticos. En ambos países pueden contarse por decenas de miles los asesinados, por centenas de miles los desplazados y refugiados, por millones los amenazados. Guatemala y El Salvador represettan hoy una de las fronteras más dramáticas no sỏlo de los derechos humanos sino de la humanidad misma. En ambos paises de ha hablado técnicamente de genocidio, 1o cual debe golpear la conciencia de todos los hombres. pero especialmente de los latinoamercanos y de los españoles. La situación es distinta en ambos palses, sobre todo porque pudiea pensarse que en El Salvador se estå más prỏximo a un principio de solución no porque hayan mejoradoz los sentimientos humanitarios de los principales responsables de la represion sino porque las condiciones objetivas están ya llevando a la necesidad de un "basta ya" de guerra y de represión. Hay posibilidad de terminar con una situación intolerable y esto, además de desperatar esperanza, agui jonea la conciencia individual y colectiva para ayudar a que pronto vuelva la paz y, al menos, un respeto básico a las vidas humanas. Para ello no es tâ de más hacer algunas reflexiones que aclaren la peculiaridad de la situación salvadoreña, lo cual sirve también de algún modo para aclarar la situación guatema1teca.

La raiz profunda de la violación de los derechos humanos en la zona es la violencia originarla que representa la injusticia estructura1. Juan Pablo II en un Mensaje dirigido a la Congerencia Epsicopal de El Salvador el 6 de Agosto de 1982 esribia: "Me doy perfectamenee cuenta de que las discordias y divisiones que turban todavía vuestro pais y causan nuevos conflictos y violencts, encuentran su raiz verdadera $y$ profunda en las situaciones de injusticia social". E1 presidente de Colombia Betancur acaba de decirle a Reagan que la causa ůltima de las 30.000 tumbas -son ya más de 35.000-abiertas en suelo salvadoreño se deben a "hogueras prendidas por la injuseicia

social o por manos extrañas en tales zonas". Frente a esta tesís, compartida por el pueblo y las Iglesias de Estados Undios, Reagan sigue hablando incorregiblemente de "agentes de 1a inestabilidad (que desean imponer nuevas formas de imperialismo y tirania" como dijo en San José o de "insurrección apoyada por fuereas extrañas" camo dijo 
en Bogotá. Evidentemente Juam Pablo II y Betancur tienen muchísima mayor verdad en sus palabras que Reagan, a quien no le interesa preguntarse por la causa última de la situación, con lo cual no puede enrender la respuesta que los movimientos politico-militares han pretendido dar en los ủltimos tres años, una vez agotados los medios pacificos de tipo politico-electoral.

Efectivamente a esta injusticia estructural tanto en Guatemala como en El Salvador se ha respondido con una combinación de violencia armada -verdaderos ejêrcitos populares que combaten con los ejërcitos ofi= ciales-y de presión política tanto a través de organización de masas como de actividad de partidos politicos en la clandestinidad. El elemento determinante en esta respuesta lo ha constituido ksarsxamar desde Enero de 1981 la violencia armada, que se ha tratado de impedir en El Salvador -de la misma forma que en Guatemala-por la via del enfrentamiento militar como, sobre todo, por la via de la masiva y sistemátiva violación de los derechos humanos. El embajador de Estados Unidos en El Salvador, Hinton, decía en un discurso tenido el 29 de Octubre de 1982: "desde 1979, tal vez unos 30.000 salvadoreños hans sido asesinados, no muertos en batalla", pero asesinados -la palabra es del embajador- por quienes están actualmente en el poder, que por 10 mismo sỏlo en 200 casos han podido ser condenados y siempre con condenas leves. El Papa se ha referido a este mismo punto con extraordinaria clarividencia y valentías "La metodología de la violencia que ha llevado a una guerra fratricida, -situando a un lado a cuantos cons 1 deran la lucha armada como un instrumento necesario para conseguir un nuevo orden social, y al otro lado a cuantos recurren a los principios de la seguridad nacional para legitimar represiones brutales-, no en cuentra una justificación racional, y mucho menos cristiana". E1 Papa no está en favor de la solución violenta y busca la feconciliación entre las dos partes en conflicto, pero esto no obsta para que distinga entre quienes han ido a la lucha armada en busca de un nuevo orden social que termine con la injusticia estructural y los que ñan ido a la represión brutal amparados en la doctrina de la seguridad nacional.

Nos encontramos asi con un esquema claro: injusticia estructura1resistencia activa a esa injusticia-represión aterrorizadora que im posibilite el que las masas se unan a quienes en su nombre luchan con tra la injusticia esgructura1. Es probable que si no hubiera esa resistencla ätiva las violaciones de los derechos humanos no serian tan masivas y brutales, pero es evidente que la llamada lucha contrainsur. 
gente no legitima esa masa de asesinatos y el modo mismo de conducir la guerra, de la que el presidente de Guatemala, Rios Montt, ha dicho que no es de tierra quemada sino de comunistas quemados. A este esquema se superpone otro, el del aniquilamiento, propiciado por Estados Unidos de todas aquellas fuerzas que en el årea no se le sometan $y$, sobre todo, de aquellas fuerzas que pudiexan simpatizar con Nicaragua, Cuba y la Unioon Soviêtica. Estados Unidos se allnea asl con quienes someten el respeto a los derechos humanos fundamentales, incluido el derecho a la vida, a la seguridad nacional.

Estados Unidos "querria" una mejora paulatina en el respeto a 10 los derechos humanos, pero siempre que esa mejora no llevara consigo un fortalecimiento de los movimientos revolucionarios. Esta tesis es fundamental en la politica exterior de Estados Unidos y probablemente en el de cualquier superpotencia. En esea dialéctica entre 1o que querría y lo que qui ere está la clave para entender y valorar 10 que de mejoría puede haber en el respeto de los derechos humanos en El Salvador durante 1982, porque en Guatemala no puede hablarse ni siquiera de mejoria eparente, por 10 menos en lo que se refiere a la población indigena y campesina. ¿Ha habio detioría en el respeto a los derechos humanos por lo que toca a E1 Salvador durante 1982, como 10 certificó la Adminstraciỏn Reagan en Agosto pasado y lo volverå a hacer en el próximo Enero? Esta pregunta es importante no para legitimar la ayuda norteamericana que de todas ma. neras se va a dar sino para sroguruxwis saber si por este camino de la mejoría paulatina se puede resolver el problema de los derechos humanos en EI Salvador.

Hay, sin duda, datos que permiten hablar de mejoria. Frente a los más de 12,000 asesinatos que hubo en 1981, en 1982 desde Enero al 15 de Noviembre se han contabilizado 4.168 y 923 capturados y/o desaparecidos. Se ha avanzado en los juicios contra los miembers de los cuerpos de seguridad responsables de loa asesinatos de las religiosas y de los asesores norteamericanos. Se ha iniciado algún saneamiento de los cuerpos de seguridad en busca de un mayor cantrol de los escuadrones de la muerte $y$ parece irse buscando un mayor control de las conexiones de la extrema derecha con el conjunto de la Fuerza Armada. Se ha establecido sauna Comisión de Derechos

Humanos oficlal No ha habido asesinatos de altos dirigentes politi$\cos$ y religiosos, aunque ha habido "desaparecimientos" de importan- 
cés lideres sindicales y capturas por "hombres fuertemente armados vestidos de civil" de quienes representaban en el interior del pais al Frente Democrâtica Revolucionario (FDR). Tambiên es apreclable en parte del Gobierno y en parte de la Fuerza Armada un nue vo estilo que busca ceñirse mâs a la ley pretende evitar villaciones públicas de los derechos humanos.

Pero todo ello no debe oscurecer el punto central. Sigue habiendo en E1 Salvador una cuota intolerable de violación de los derechos humanos y no caba acudir a la coartada de la "mejoría" como si la gravedad hubiera pasado. La "mejoria" puede ser pretexto para 1a certificación Reagan, puedo incluso estimarse cmo síntoma de que las cosas pueden entran por otra via, pero no constituye justificacioon alguna. Resulta, en efecto, intolerable que en un año mue ran aseshinados mås de cinco mil ciudadanos aiviles inefensos -cantí dad que supondría proporcionalmente en España, más de treinta cị̂ co mil asesinados en un años-, sobre todo si han de sumarse a los más de treinta mil reconocidos por el propio embajador norteamericano. No se ha cambiado de esquema y lo que puede verse es una uti 1ización más racional del mismo, pero el esquema sigue sien do intolerable. Este esquema consiste en hacer parte insustituible de la lucha contrainsurgente la represión aterrorizadora del pueblo. Lo que ha mejorado es el cálculo: "ya" no es necesario asesi. nar a quince mil para conseguir el efecto deseado basta con chnco mil, pero si fuera necesario aumentar la cifra el aparato represivo está preparado para hacer10. La realidad es mảs compleja de 10 que aqui expongo, pero la línea fundamental es correcta. No todos los dirigentes participan en ella, pero sigue siendo una ley que impone su fuerza macabra a voluntades que no desearian seguir ese camino. En Guatemala las cosas andan muchop peor y cada vez se extreman las medidas represivas y se hace de ellas un proceso sistemåtico de aniquilación. El inacapeable acercamiento a la zona de los mêtodos israelíes y de los måximos responsables del gencidio palestino hace pensar lop peor.

Sölo si hubiera una subordinación absoluta y permanente de cualquier proyecto político al respeto de los derechos humanos; soblo si la violación de los derechos humanos deja de ser parte sustancial del proyecto político-militarı sólo si comienza el desmante. lamientoxx sistemático del aparato represivo, podría aceptarse que se da en realidad una mejoría. ¿Es esto posible en E1 Salvador? 
Esto es posible si se termina con la guerra, con el estado de guerra, lo cual es hoy más posíble que nunca. Y esta guerra no puede terminar por el camino de la vicooria miliaar sino soblo por el camino del diălogo y de la negociación, que hoy se presetan más realistas que nunca, precisamenee porque la guerra no sỏlo lleva meses y meses sin logro ninguno para la Fuerza Armada y porque en los meses de octubre y de noviembra ha demostrado una inesperada potencia nueva por parte del FMLN. Pues bien, ahora que el FMLN ha demostrado una vez más su cada vez mayor potencia militar es cuando reltera su oferta de diálogo, presentada por dos de los sels obispos salvadoreños al Gobierno y al Alto Mando y no mal recibida por estos, ni siquiera por la Asamblea Constotuyente que cangeló la propuesta de rechazar toda suerte de diálogo, tal como los pretendía la facción más extremista de la derecha. La semilla del diălogo entre las partes involucradas en el conflicto parece haber caido esta vez en buena tierra. aunque sus posibilidades no dejan de ser escasas. Si ella no proppera, continuará la guerra, contínuarå el sabota je que está estrangulando la economía del país, seguirâ sobre todo la represión y la permanente violación de los derechos humanos.

Es ahora cuando deben hacerse más presentes las fuerzas internacionales que quieren la paz y el derecho para la regiôn centroamericana. Si Estados Unidos e Israel se meten en * el årea, es tiempo de que tambiēn se hagan presente en ella fuerzas que tienen real peso hisfifrico en ella y justos ineereses humanos que propiciar. La responsabilidad de Espa角 y de su nuebo Gobierno son grandes, porque es mucho lo que puede hacer y porque puede representan la cabeza de una presencia mås amplia de naciones que busquen la paz y el desarro110 del årea centroamericana. Venezuela y Colombla junto con México estân cada vez mås cerca de la tesis del diålogo y de la cooperación España con otros palses europeos de gobiernos socialistas pueden representar el "término medio" entre las demandas de los gobiernos centroamericanos derechistas y las de los movimientos revolucionarios. Con ello no harian sino contrarrestar orros influjos perniciosos y permitir a los pueblos centroamericanos el cese de la violencia, el

principio de su autodeterminación y la lucha contra aquella injusticía estructural que están en la raíz de todos los males, como acaba de recordarlo Juan Pablo II, que se harả presente en el próximo mes de Febrero para anunciar una paz fundada en la justicia y en la libertad, en las armas del diálogo frente a las armas de la violencia. 